\title{
Road Detection from High Satellite Images Using Neural Networks
}

\author{
Idris Kahraman, Muhammed Kamil Turan, and Ismail Rakip Karas
}

\begin{abstract}
In this paper, we propose a road detection model approach based on neural networks from satellite images. The model is based on Multilayer Perceptron (MLP) which is one of the most preferred artificial neural network architecture in classification and prediction problems. According the neural network, the RGB values are used for deciding the pixel belongs to road or not. The found road pixels are marked in the output image.
\end{abstract}

Index Terms-Road detection, road extraction, neural networks.

\section{INTRODUCTION}

\section{A. Road Extraction}

Satellite images can assist the planning and coordination of global change research by facilitating the design of research strategies and the implementation of methodologies. The presence of high resolution satellite images and their potential to be used in wide variety of applications such as preparing and updating maps have made the extraction of object, especially roads and buildings, a new challenge in remote sensing. Using image processing for analyzing and extracting information from high resolution satellite imagery to create feature extraction.

Roads are one of the most important manmade objects in map production from satellite images that must be extracted accurately. Acquisition and updating geospatial information is characterized by a large amount of manual work, and is thus rather costly and slow. Research and development have investigated new and effective solutions. Road extraction provides means for creation, maintaining, and updating transportation network. It also provides data bases for traffic management, automated vehicle navigation and guidance [1].

\section{B. Related Researches Review}

A road detection strategy based on the neural network classifiers was introduced by Mokhtarzade and Valadan, where a variety of input spectral parameters were tested on the functionality of the neural network for both road and background detection [1].

It is proposed a new fuzzy segmentation method for road detection in high resolution satellite images with only a few number of road samples. Afterward by using an advanced mathematical morphological operator, road centerlines were extracted [2].

Manuscript received April 15, 2015; revised July 23, 2015.

Idris Kahraman is with the Karabuk University, Turkey (e-mail: idriskahraman@karabuk.edu.tr).
A shoreline detection strategy based on the neural network classifiers was introduced by Turan, 2012. Their algorithm detects the shoreline between land and sea with the $90.03 \%$ success rate [3].

It is proposed a method for detection road junctions in aerial images artificial with neural networks. The paper describes an approach of automatic junction detection using raster and vector information: mean and standard deviation of gray values, edges as road borders etc. The derived feature set was used to train a feed-forward neural network, which was the base of the junction operator. The operator decides for a running window about having a road junction or not [4].

\section{Structure of the Used Artificial Neural NETWORK}

Neural Networks are made up of simple processing units called nodes or neurodes. The main task associated with a neurode is to receive input from its neighbours (the output of other neurodes), compute an output and send that output to its neighbours [5].

Neurodes are usually organized into layers with full or random connections between successive layers. There are three types of layers: input, hidden and output layers in charge of receiving, processing and presenting the final results respectively.

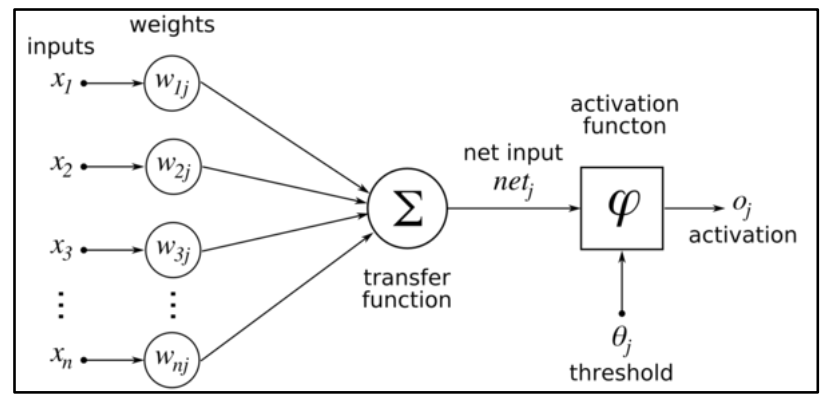

Fig. 1. A node of MLP: an artificial neuron.

Road detection from satellite images can be considered as a classification process in which pixels are divided into road and background classes. Recent researches have shown ANNs to be capable of pattern recognition and classification of image data. For using neural networks in road detection, input layer is consisted of neurodes the same number as input parameters and output layer is made up of just one neurode that shows the belief of network whether the input parameters can represent a road pixel or not. Usually one hidden layer is sufficient, although the number of neurodes in the hidden layer is often not readily determined [6]. More neurodes in hidden layer enables the network to learn more 
complicated problems, but there would be an associated increase in training time [7].

In this study we have used Multilayer Perceptron (MLP) as a neural network structure. One of the most frequently used neural network architectures in classification is MLP. MLP consists of a network of nodes arranged in layers. The general structure of MLP consist of three or more units arranged in layers of processing nodes: an input layer that receives external inputs, one or more hidden layers and an output layer that produces the classification results. Each node in MLP can be modelled as an artificial neuron (Fig. 1.) [8]

In the MLP, each neuron $\mathrm{j}$ in the hidden layer computes the sum of input $\mathrm{x}_{\mathrm{i}}$ weighted by respective connection weight $w_{i j}$ and calculates its output $y_{j}$ as a function of the sum.

$$
y_{i}=f\left(\sum W_{j i} X_{i}\right)
$$

where $f$ is the activation function which transforms the weighted sum of all signals to a neuron as multiplier. Here, activation function $(f)$ can be a simple threshold function or a sigmoidal, hyperbolic tangent, or radial basis function. The sum of squared differences between desired and actual values of output neuron e is defined as follows:

$$
e=\sum_{j}\left(y_{d j}-y_{j}\right)^{2}
$$

where $y_{d j}$ and $y_{j}$ are the desired and actual value of output neuron $j$ respectively. Each weight $w_{i j}$ is tuned to reduce the value $e$ immediately. How $w_{i j}$ is tuned depends on the training algorithm adopted [9].

\section{Generating Training DAta And Training PRocess}

In training process, it is necessary to create own training data. For this reason, according to our neural network we have 27 inputs and 1 output and training data is get manually by selecting a pixel from the image Fig. 2. For selected point, $R, G, B$ values of every neighboring pixel like $3 \times 3$ matrix, is stored. These 27 data is the input. The output is 1 or 0 (road or not road). In order to get uniform distribution we have used 20 different satellite images and we have created 650 records.

$\mathrm{R}, \mathrm{G}, \mathrm{B}$ values is range from 0 to 255 . When numeric values are normalized, neural network training is often more efficient, which leads to a better predictor. The program normalizes R, G, B values by computing with the formula. After normalizing the RGB values between 0 and 1 , is simply entered to the network as its input parameters.

$$
\operatorname{Normalized}\left(e_{i}\right)=\frac{e_{i}-E_{\text {min }}}{E_{\text {max }}-E_{\text {min }}}
$$

The network with low number hidden neurodes is very unstable which means networks functionality is highly dependent on initial weight assignment and results vary in multiple implementations. Larger hidden layer size enables the network to modulate more complicated problems, but in this case classification problem does not seem so complex since the results are quite the same for different networks. It is shown on Table I that low or high number of hidden layer decreases the success rate. For this reason some iterations should be made for determining the optimal number of hidden neurons.

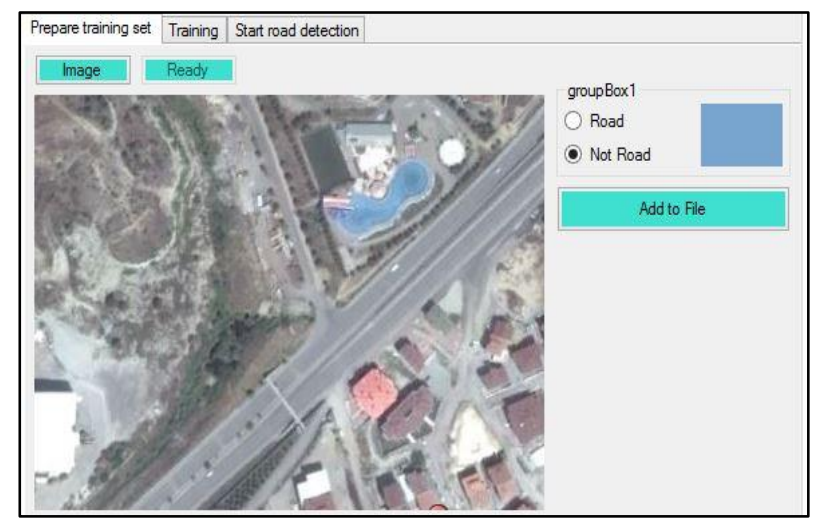

Fig. 2. Interface of creating training data.

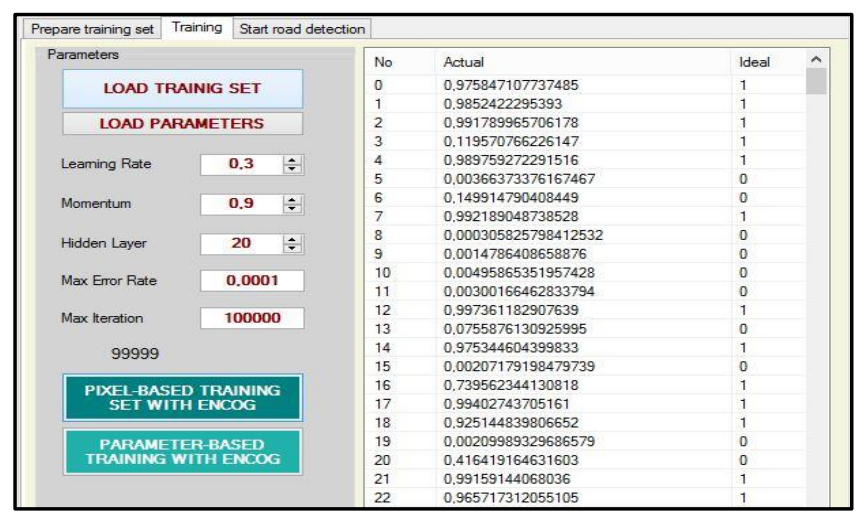

Fig. 3. Training phase and training results.

TABLE I: THE PERCENTAGE OF SUCCESS ACCORDING TO DIFFERENT MLP PARAMETERS

\begin{tabular}{|l|c|c|c|c|c|c|}
\hline Learning Rate & 0.3 & 0.4 & 0.3 & 0.3 & 0.3 & 0.2 \\
\hline Momentum & 0.9 & 0.9 & 0.9 & 0.9 & 0.9 & 0.9 \\
\hline $\begin{array}{l}\text { Input Layer } \\
\text { Neuron }\end{array}$ & 27 & 27 & 27 & 27 & 27 & 27 \\
\hline $\begin{array}{l}\text { Hidden Layer } \\
\text { Neuron }\end{array}$ & 20 & 20 & 15 & 12 & 10 & 12 \\
\hline Success & 88.25 & 87.02 & 90.88 & 93,35 & 90.57 & 87.17 \\
\hline
\end{tabular}

In our training process we have used 650 randomly shuffled records. MLP is designed in three layers including an input layer, two hidden layer and one output layer. In the input layer has 27 neurons and hidden layer has 12 neurons. In the output layer we have one neuron representing road or not. We have used backpropagation algorithm with adjusted training parameters (momentum and learning rate) as the training method and used sigmoid activation function in all layers of MLP shown on Fig. 3. For training iteration, maximum epoch number is 100.000 and maximum error 
rate 0.0001 .

In order to find optimal parameters for training phase, we vary the MLP parameters. Momentum is 0.9 , learning rate 0.2 to 0.5 . The best result have been obtained when the learning rate is set to 0.3 , momentum is set to 0.9 and hidden layer neuron is set to 12 . In the study, best prediction result has been found as $93.35 \%$ (Table I).

\section{IMPLEMENTATION RESULTS OF ROAD DETECTION}

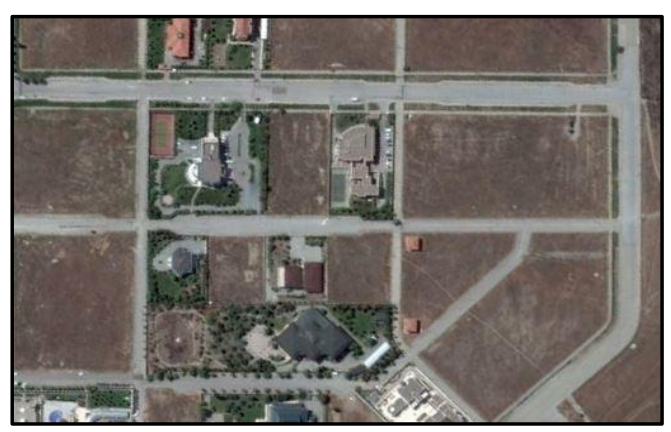

Fig. 4(a). Satellite image.

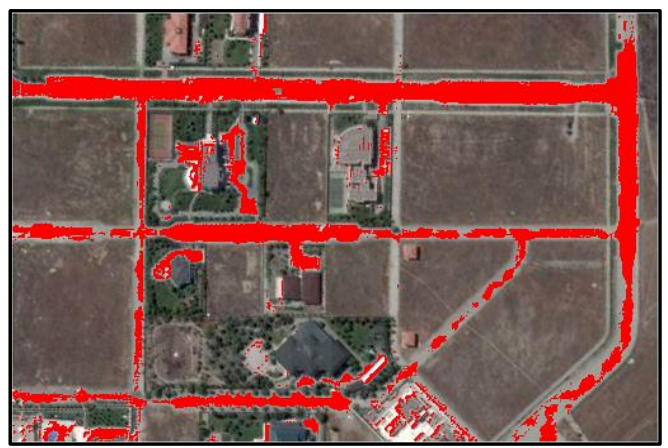

Fig. 4(b). After ANN implementation.

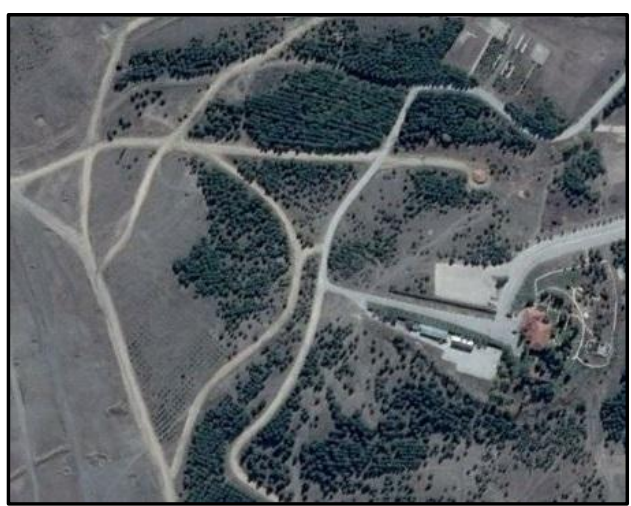

Fig. 5(a). Satellite image.

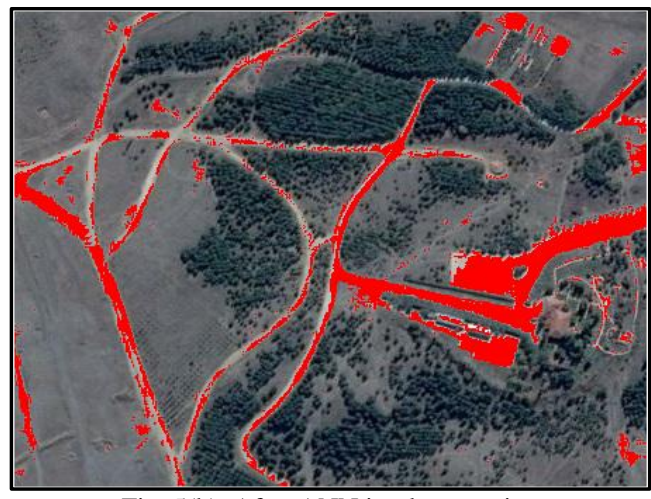

Fig. 5(b). After ANN implementation
Road detection was performed using an artificial neural network consisting of 27 neuron in its input layer in charge of receiving 3 spectral values $(R, G, B)$ as explained in section 2. The hidden layer was made up of 12 neurons and the output layer, having only one neuron, was designed to show the response of neural network. After 100.000 iterations in training phase, the results of the images that have not used in the training is showed in Fig. 4 and Fig. 5.

This data set is used only for testing the final solution in order to confirm the actual predictive power of the network. For this reason, 25 satellite images have used for generating training data. We evaluate the network by creating test data that has no data belongs to training data. The left side images (Fig. 4(a) and Fig. 5(a)) show the satellite image taken from google earth. Right side images (Fig. 4(b) and Fig. 5(b)) depicts the output of the proposed neural network structure. Almost all of the roads have been detected.

\section{CONCLUSION}

The main objective of this research was to develop an automatic method to extract rural and sub urban roads using neural network algorithm. It is successfully developed a semi-automatic method to extract roads from very high resolution satellite images by using learning vector quantization neural network algorithm. In this article the impact of input parameters on neural network's ability for road detection from high resolution satellite images was tested on google earth images.

According the nature of the problem, number of classes given by the developed software were observed considered relatively sufficient. In this study, some region images was expected to be classified as road or not. This expectation has been met percent $93.35 \%$ availability. The algorithm is implemented on two satellite images that are not used in training phase. The developed algorithm is successful for detecting road in a region.

Our future plan is the addition of more parameters for classifying the satellite images. Detection of objects such as buildings, road segments and road junctions, and urban area boundaries play crucial roles especially for municipalities, government agencies, rescue teams, military, and other civil agencies.

\section{REFERENCES}

[1] M. Mokhtarzdae and M. J. V. Zoej, "Road detection from high resolution satellite images using artificial neural networks," International Journal of Applied Earth Observation and Geoinformation, vol. 9, no. 1, pp. 32-40, 2007.

[2] A. Mohammadzadeh, A. Tavakoli, and M. J. V. Zoej, "Road extraction based on fuzzy logic and mathematical morphology from pan-sharpened IKONOS images," The Photogrammetric Record, vol. 21, no. 113 , pp. 44-60, 2006

[3] M. K. Turan and I. R. Karas, "Determination of the shoreline by using back propagation artificial neural networks," in Proc. the 4th Remote Sensing and GIS Symposium (UZAL-CBS '2012), Oct 16-19, 2012, Bulent Ecevit University, Zonguldak, Turkey.

[4] A. Barsia and C. Heipkeb, "Artificial neural networks for the detection of road tunctions in aerial images," ISPRS Archives, Part 3/W8, Munich, 17.-19. Sept. 2003

[5] G. Y. Yang, "Geological mapping from multi-source data using neural networks," MSc Thesis, University of Calgary, Canada, 1995.

[6] J. A. Richard, Remote Sensing Digital Image Analysis: Introduction, Second Edition, Springer, ISBN 0-387-5480-8, New York, 1993. 
[7] G. M. Foody, M. B. McCulloch, and W. B. Yates, "Classification of remotely sensed data an artificial neural network: Issues related to training data characteristics," Photogrammetric Engineering and Remote Sensing, vol. 61, no. 4, pp. 391-401, 1995.

[8] H. Yan, Y. Jiang, J. Zheng, C. Peng, and Q. Li, "A multilayer perceptron-based medical decision support system for heart disease diagnosis," Expert Systems with Applications, vol. 30, no. 2, pp. 272$281,2006$.

[9] E. D. Ubeyli and I. Guler, "Detection of electrocardiographic changes in partial epileptic patients using Lyapunov exponents with multilayer perceptron neural networks," Engineering Applications of Artificial Intelligence, vol. 17, no. 6, pp. 567-576, 2004.

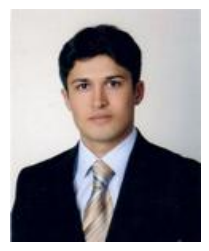

Idris Kahraman was born in Bolu / Turkey in 1985. He received his bachelor degree in system engineering from Military Academy in Ankara, Turkey, in 2007. $\mathrm{He}$ gets his master degree in computer engineering from Karabuk University in 2014. He studies in geographical information system.

He has been working in Karabuk University since 2010 as a research assistant in computer Engineering department, Karabuk University which is located on west part of blacksea Region of Turkey. 